\title{
Análise do uso de medicamentos psicotrópicos no sistema penitenciário do Estado do Ceará
}

\author{
Analysis of the Use of Psychotropic Medications in the Penitentiary System of the State of \\ Ceará
}

Análisis del Uso de Medicamentos Psicotrópicos en el Sistema Penitenciario del Estado de Ceará

\section{Allyanne Peixoto de Almeida ${ }^{1}$ Renan Pereira De Lima² Arlandia Cristina Lima Nobre de Morais $^{3}$}

RESUMO. Objetivo: Avaliar a utilização de medicamentos psicotrópicos no sistema penitenciário. Metodologia: Os dados foram obtidos através de levantamento prospectivo, observacional, quantitativo e descritivo. Foram analisados 30 prontuários de indivíduos condenados por sentença judicial, coletados no Instituto Psiquiátrico Governador Stênio Gomes localizado no município de Itaitinga-CE no período de julho a setembro de 2012. O projeto foi aprovado pelo Comitê de Ética em Pesquisas da Universidade de Fortaleza sob parecer $n^{\circ}$ 258/2011. Resultados: o perfil demográfico aponta para pacientes do gênero masculino, solteiro, analfabetos e fumantes. O medicamento mais utilizado no tratamento dos penitenciários com transtornos mentais foi o haloperidol (27,5\%). Observou-se 58 potenciais interações medicamentosas (1,7 $\pm 1,85$ interações por pacientes). Quanto à classificação das interações, $22,4 \%$ do tipo menor, $51,7 \%$ do tipo moderada e $24,13 \%$ do tipo maior, $74,10 \%$ com início demorado, 24,13\% com início rápido, de acordo com a documentação na literatura 41,36\% suspeita, 3,44\% estabelecida e 53,43\% possível. Dos transtornos mentais diagnosticados 40,1\% apresentam Esquizofrenia paranoide, residual e não específica. A prática de homicídio foi atribuída a $29,91 \%$ dos apenados esquizofrênico, na modalidade qualificada. Outros delitos envolvendo a violência apresentaram estreita relação com este transtorno mental. Conclusão: Os pacientes esquizofrênicos foram responsáveis pela prática de homicídios em taxas maiores que aqueles que possuem outro transtorno mental e a conduta agressiva dos pacientes esquizofrênicos parece ter sido uma variável importante da prática de crimes envolvendo a violência.

Palavras-chave: Psicotrópicos. Transtornos mentais. Crime. Interações Medicamentosas.

ABSTRACT. Objective: To evaluate the use of psychotropic drugs in the penitentiary system. Methodology: Data were obtained through a prospective, observational, quantitative and descriptive survey. We analyzed 30 medical records of individuals convicted of a judicial sentence, collected at the Governador Stênio Gomes Psychiatric Institute located in the city of Itaitinga-CE from July to September 2012. The project was approved by the Research Ethics Committee of the University of Fortaleza, No 258/2011. Results: The demographic profile points to male patients, single, illiterate and smokers. The most commonly used medication in the treatment of penitentiaries with mental

\footnotetext{
${ }^{1}$ Farmacêutica, Centro de Ciências da Saúde

${ }^{2}$ Mestrando em Farmacologia na Universidade Federal do Ceará

${ }^{3}$ Farmacêutica, doutora em farmacologia, Advogada, Professora titular, Curso de farmácia, Universidade de Fortaleza
} 
disorders was haloperidol (27.5\%). There were 58 potential drug interactions $(1.7 \pm 1.85$ interactions per patient). As for the classification of the interactions, $22.4 \%$ of the minor type, $51.7 \%$ of the moderate type and $24.13 \%$ of the major type, $74.10 \%$ with a delayed onset, $24.13 \%$ with a rapid onset, according to documentation in the literature $41.36 \%$ suspected, $3.44 \%$ established and $53.43 \%$ possible. Of the mental disorders diagnosed, $40.1 \%$ present paranoid schizophrenia, residual and non-specific. The practice of homicide was attributed to $29.91 \%$ of the distressed schizophrenic, in the qualified modality. Other crimes involving violence were closely related to this mental disorder. Conclusion: The schizophrenic patients were responsible for homicide at rates higher than those who had another mental disorder and the aggressive behavior of schizophrenic patients seems to have been an important variable in the practice of crimes involving violence.

Keywords: Psychotropic drugs. Mental disorders. Crime. Drug Interactions.

RESUMEN. Objetivo: Evaluar la utilización de medicamentos psicotrópicos en el sistema penitenciario. Metodología: los datos fueron obtenidos a través del levantamiento prospectivo, observacional, cuantitativo y descriptivo. Se analizaron 30 prontuarios de individuos condenados por sentencia judicial, recogidos en el Instituto Psiquiátrico Gobernador Stênio Gomes ubicado en el municipio de Itaitinga-CE en el período de julio a septiembre de 2012. El proyecto fue aprobado por el Comité de Ética en Investigaciones de la Universidad de Fortaleza bajo opinión $n{ }^{\circ}$ 258/2011. Resultados: El perfil demográfico apunta a pacientes del género masculino, soltero, analfabetos y fumadores. El medicamento más utilizado en el tratamiento de los penitenciarios con trastornos mentales fue el haloperidol (27,5\%). Se observaron 58 posibles interacciones medicamentosas $(1,7$ $\pm 1,85$ interacciones por pacientes). En cuanto a la clasificación de las interacciones, el $22,4 \%$ del tipo menor, el $51,7 \%$ del tipo moderado y el $24,13 \%$ del tipo mayor, el $74,10 \%$ con início demorado, el $24,13 \%$ con inicio rápido, documentación en la literatura $41,36 \%$ sospechosa, 3,44\% establecida y 53,43\% posible. De los trastornos mentales diagnosticados $40,1 \%$ presentan esquizofrenia paranoide, residual y no específica. La práctica de homicidio fue atribuida al 29,91\% de los apenados esquizofrénicos, en la modalidad calificada. Otros delitos involucrando la violencia presentaron estrecha relación con este trastorno mental. Conclusión: Los pacientes esquizofrénicos fueron responsables de la práctica de homicidios en tasas mayores que aquellos que poseen otro trastorno mental y la conducta agresiva de los pacientes esquizofrénicos parece haber sido una variable importante de la práctica de crímenes involucrando la violencia.

Palabras-Ilave: Psicotrópicos. Trastornos mentales. Crimem. Interacciones medicamentosas.

\section{Introdução}

A assistência à saúde aos indivíduos privados de liberdade era oferecida de forma residual e parcial, com o objetivo de suavizar os problemas frequentes como doenças sexualmente transmissíveis (DST), síndrome da imunodeficiência adquirida (AIDS) e imunizações, esquecendo ainda as condições de insalubridade que geravam sérios prejuízos à saúde dos penitenciários (1). 
Existe também o desacato aos direitos humanos dos apenados devido à falha na educação, carência de profissionais qualificados, selas lotadas, condições precárias para sobreviver. É necessário que os penitenciários possam usufruir também dos direitos à saúde, inclusive psíquica, já que "à saúde é direito de todos e dever do Estado" conforme prevê o artigo 196 da Constituição Federal de 1988 (CF/88) (2).

Diante disso, o Ministério da Saúde (MS) e o Ministério da Justiça (MJ) são destinados a promover a atenção integral à saúde do indivíduo preso. Ressalta-se que promover a saúde do indivíduo privado de liberdade não só traz benefícios a este, mas também melhoria às condições de saúde e segurança no local de trabalho para os profissionais do sistema penitenciário. Portanto, melhorar as condições que atingem negativamente a saúde destas pessoas, tais como superpopulação, alimentação precária e falta de atividades ocupacionais, contribui para a diminuição das tensões e da violência dentro da penitenciária (1).

Os apenados apresentam as mais variadas doenças no interior das prisões. As mais habituais estão relacionadas ao aparelho respiratório. Também são comuns hepatite e a síndrome da imunodeficiência adquirida. Segundo Assis (3), aproximadamente 20\% dos presos brasileiros são portadores de HIV; o que torna uma preocupação a mais no âmbito de saúde pública. Há também outro problema que se destaca no ambiente prisional: as doenças psiquiátricas. Nessa população são encontrados transtornos de personalidade e os vícios (4).

Os manicômios (hospitais psiquiátricos) recebem os internos, que são doentes com transtornos mentais que cometeram crimes, buscando garantir a reintegração social. O que se percebe, no entanto, é que esses criminosos são isolados da sociedade para manter a "segurança", já que são consideradas pessoas "anormais" (5).

No sistema penitenciário a assistência farmacêutica compreende: seleção de medicamentos; programação e aquisição de produtos farmacêuticos; recebimento e armazenamento; distribuição; prescrição e dispensação, inclusive de medicamentos psicoativos. As farmácias das penitenciárias deveriam possuir farmacêuticos e auxiliares de farmácia para desempenhar essas atividades (6). Todavia, o que se apresenta na realidade é um número insuficiente de profissionais, o que impede a eficiência da assistência farmacêutica. 
Há vários fatores que contribuem para o alto consumo de drogas psicotrópicas nas penitenciárias, como aumento da prevalência de transtornos mentais nas prisões, destacando-se a esquizofrenia; as características de funcionamento do sistema de saúde prisional, incluindo a acessibilidade para consultas médicas e as maiores possibilidades de um ambiente fechado, de detecção e monitoramento; co-morbidade muito elevada de abuso de substâncias que condiciona uma demanda excessiva desses medicamentos e pode resultar em receitas para a tolerância associada não só com um conflito médicopaciente, mas também com o nível geral de tensão entre os internos (7).

A esquizofrenia é um transtorno de caráter crônico caracterizado pela presença de comportamento psicótico, podendo levar a graves prejuízos para seu funcionamento individual, familiar, profissional e social. O paciente com esquizofrenia apresenta sintomas, tais como delírios e alucinações que podem apresentar-se como atos violentos, agressões verbais e físicas (8).

Diante dessa situação e da elevada demanda por medicamentos psicotrópicos, surge à preocupação a cerca do controle do uso de tais substâncias. Os psicotrópicos são substâncias que podem determinar dependência física e/ou psíquica e são medicamentos sujeitos ao controle especial. A Portaria n. 344/98 controla a prescrição e dispensação dos psicofármacos, antiretrovirais, retinóides de uso sistêmico, imunossupressores e anabolizantes. Este estudo tem importância social e analisará a utilização de medicamentos psicotrópicos pelos apenados, avaliando assim a presença de problemas relacionados ao uso desses medicamentos e se há uma relação entre a doença e o crime cometido.

\section{Metodologia}

O estudo foi um levantamento prospectivo, observacional, quantitativo e descritivo em 30 prontuários de indivíduos condenados por sentença judicial, com o objetivo de avaliar o uso de psicotrópicos. Os dados foram coletados no Instituto Psiquiátrico Governador Stênio Gomes, localizado no município de Itaitinga-CE (região metropolitana de Fortaleza), no período de julho a setembro de 2012. Foram utilizados os prontuários dos presos que cumprem pena igual ou superior a 4 (quatro) anos de reclusão, que apresentavam transtornos mentais e façam uso de psicotrópicos, mediante consentimento através da assinatura no Termo de Fiel Depositário e no Termo de Consentimento Livre e Esclarecido, 
pelo responsável pelo serviço e paciente, respectivamente. O projeto foi submetido e aprovado pelo comitê de ética em pesquisas da Universidade de Fortaleza sob parecer $n^{\circ}$ 258/2011.

Os dados foram analisados por meio do EPI-info versão 3.3.2. Essa análise constitui em descrever as características gerais da amostra com relação às variáveis sociodemográficas, clínicas (doença de base, posologia, duração do tratamento, presença de co-morbidade, uso de outros medicamentos) e os crimes cometidos, obtendo a média, desvio-padrão e frequência relativa. Os medicamentos foram classificados de acordo com AnatomicalTherapeuticChemical (ATC) e as interações segundo início, severidade e documentação (9).

\section{Resultados}

A amostra foi composta por 30 prontuários de sujeitos que cumprem pena judicial com idade média de 42,46 9,91anos, variando de 24 a 66 anos, sendo apenas 1 idoso e todos do gênero masculino e fumantes. Os registros nos prontuários sobre o estado conjugal revelaram que $20 \%$ dos apenados são casados; $76,7 \%$ solteiros e $3,3 \%$ viúvos.

Com relação ao nível de escolaridade, os dados analisados mostraram que 43,3\% dos apenados não frequentaram a escola, considerados analfabetos, superando o total dos que não concluíram o ensino fundamental. Apenas 6,7\% possuíam o ensino superior completo. A tabela 1 demonstra a distribuição sociodemográfica da população carcerária. 
Tabela 1. Características Sociodemográficas dos Pacientes em Estudo.

\begin{tabular}{lcccc}
\hline & & $f$ & $\%$ & IC - 95\% \\
\hline Faixa etária & $20-30$ & 3 & 10 & $2,1-26,5$ \\
& $31-40$ & 10 & 33,3 & $17,3-52,8$ \\
& $41-50$ & 11 & 36,7 & $19,9-56,1$ \\
& $>51$ & 6 & 20 & $7,7-38,6$ \\
\hline Estado civil & Casado & 6 & 20 & $7,7-38,6$ \\
& Solteiro & 23 & 76,7 & $57,7-90,1$ \\
& Viúvo & 1 & 3,3 & $0,1-17,2$ \\
\hline Escolaridade & ANALF. & 13 & $43,30 \%$ & $25,5-62,6$ \\
& EFC & 1 & $3,30 \%$ & $0,1-17,2$ \\
& EFI & 12 & $40,00 \%$ & $22,7-59,4$ \\
& EMC & 1 & $3,30 \%$ & $0,1-17,2$ \\
& EMI & 1 & $3,30 \%$ & $0,1-17,2$ \\
& NSC & 2 & $6,70 \%$ & $0,8-22,1$ \\
\hline
\end{tabular}

* ANALF: ANALFABETO; EFC: ENCINO FUNDAMENTAL COMPLETO; EFI: ENSINO FUNDAMENTAL INCOMPLETO; EMC: ENSINO MÉDIO COMPLETO; EMI: ENSINO MÉDIO INCOMPLETO; NSC: NÍVEL SUPERIO COMPLETO; S: SIM.

A tabela 2 expressa as distribuições por classe de psicotrópicos utilizados pela população carcerária em estudo. Os fármacos foram classificados de acordo com nível 5 da classificação terapêutica anatômica química (ATC-AnatomicalTherapeuticChemical). Os antipsicóticos foram a classe de psicotrópicos mais prescritos, destacando-se o Haloperidol (27,5\%), Clorpromazina (11,5\%) e Risperidona (10,6\%), o que equivale a $41,6 \%$ de todos os psicotrópicos prescritos. No grupo dos ansiolíticos e hipnóticos, o Diazepam foi o mais prescrito com uma frequência de 20,4\%. Entre os antiepiléticos destacam-se a Clorpromazina $(4,4 \%)$ e o Ácido Valpróico (3,5\%). Com relação aos Antidepressivos, o mais prescrito foi a Amitriptilina (1,8\%). 
Tabela 2. Distribuição dos fármacos prescritos por classe de psicotrópicos

\begin{tabular}{llcccc}
\hline \multicolumn{1}{c}{ Classe dos Psicotrópicos } & \multicolumn{1}{c}{ Fármacos } & ATC & $f$ & $\%$ & IC - 95\% \\
\hline Antiepiléticos & Carbamazepina & N03AF01 & 5 & $4,40 \%$ & $1,5-10,0$ \\
& Ácido valpróico & N03AG01 & 4 & $3,50 \%$ & $1,0-8,8$ \\
& Fenobarbital & N03AA02 & 2 & $1,80 \%$ & $0,2-6,2$ \\
Ansiolíticos e hipnóticos & Diazepam & N05BA01 & 23 & $20,40 \%$ & $13,4-29,0$ \\
& Alprazolam & N05BA12 & 1 & $0,90 \%$ & $0,0-4,8$ \\
Antipsicóticos & Levomepromazine & N05AA02 & 3 & $2,70 \%$ & $0,6-7,6$ \\
& Haloperidol & N05AD01 & 31 & $27,40 \%$ & $19,5-36,6$ \\
& Clorpromazina & N05AA01 & 13 & $11,50 \%$ & $6,3-18,9$ \\
& Risperidona & N05AX08 & 12 & $10,60 \%$ & $5,6-17,8$ \\
Antiparkinsoniano & Biperideno & N04AA02 & 15 & $13,30 \%$ & $7,6-20$, \\
Antidepressivos & Amitriptilina & N06CA01 & 2 & $1,80 \%$ & $0,2-6,2$ \\
& Fluoxetina & N06AB03 & 1 & $0,90 \%$ & $0,0-4,8$ \\
Modificador do humor & Carbonato de Lítio & M01AE01 & 1 & $0,90 \%$ & $0,0-4,8$ \\
\hline
\end{tabular}
TOTAL 113

ATC = AnatomicalTherapeuticChemical/ *HaloperidolDecanoato: 19,5\%; Haloperidol Comprimido: 8\%.

Dos 30 pacientes do estudo observou-se 58 potenciais interações medicamentosas, com média de 1,7 \pm 1,85 interações por pacientes. Uma interação medicamentosa potencial pode ser prevista a partir dos conhecimentos das propriedades farmacológicas das drogas envolvidas, não ocorrendo necessariamente em todos os pacientes analisados no presídio.

Quanto à classificação das potenciais interações medicamentosas identificadas no estudo, 22,40\% das interações foram consideradas de menor gravidade; 51,70\% de gravidade moderada e, $24,13 \%$ de maior gravidade, sendo $24,13 \%$ de início rápido e $74,10 \%$ de início demorado. Quanto às documentações na literatura, 41,36\% foram classificadas como suspeitas; 3,44\% estabelecida e 53,43\% possíveis. As interações medicamentosas estão descritas na tabela 3.

Quanto ao perfil farmacocinético e farmacodinâmico, as potenciais interações foram classificadas, em sua maioria como interações farmacocinéticas. As reações químicas podem ocorrer entre um ácido e uma base fraca, resultando em sal e água, e isto pode ocasionar um aumento ou diminuição da biodisponibilidade do fármaco no sangue. 
Tabela 4. Características físico-químicas dos medicamentos utilizados pelos detentos.

\begin{tabular}{lcccc}
\hline Medicamento & Fórmula Química & Pka & pH & Absorção \\
\hline Clorpromazina & $\mathrm{C}_{17} \mathrm{H}_{19} \mathrm{CIN}_{2} \mathrm{~S}$ & 9,3 & Básico-fraca & TGl (intestino) \\
Ácido valpróico & $\mathrm{C}_{8} \mathrm{H}_{16} \mathrm{O}_{2}$ & 4,8 & Ácido-fraco & TGl (estômago) \\
Fenobarbital & $\mathrm{C}_{12} \mathrm{H}_{12} \mathrm{~N}_{2} \mathrm{O}_{3}$ & 7,3 & Base-fraca & TGl (intestino) \\
Diazepam & $\mathrm{C}_{16} \mathrm{H}_{13} \mathrm{CIN}_{2} \mathrm{O}$ & 3,4 & Base-fraca & TGl (intestino) \\
Alprazolam & $\mathrm{C}_{17} \mathrm{H}_{13} \mathrm{CIN}_{4}$ & 11,61 & Base-fraca & TGl (intestino) \\
Levomepromazine & $\mathrm{C}_{19} \mathrm{H}_{24} \mathrm{~N}_{2} \mathrm{OS} \mathrm{HC}$ & 9,4 & Base-fraca & TGl (intestino) \\
Haloperidol & $\mathrm{C}_{21} \mathrm{H}_{23} \mathrm{NCIF}_{2}$ & 8,3 & Base-fraca & TGl (intestino) \\
Clorpromazina & $\mathrm{C}_{17} \mathrm{H}_{19} \mathrm{CIN}_{2} \mathrm{~S}$ & 9,3 & Base-fraca & TGl (intestino) \\
Risperidona & $\mathrm{C}_{23} \mathrm{H}_{27} \mathrm{~N}_{4} \mathrm{FO} \mathrm{O}_{2}$ & 8,24 & Base-fraca & TGl (intestino) \\
Biperideno & $\mathrm{C}_{21} \mathrm{H}_{29} \mathrm{NO}$ & 9,25 & Base-fraca & TGl (intestino) \\
Amitriptilina & $\mathrm{C}_{20} \mathrm{H}_{23} \mathrm{~N}$ & 9,4 & Base-fraca & TGl (intestino) \\
Fluoxetina & $\mathrm{C}_{17} \mathrm{H}_{18} \mathrm{~F}_{3} \mathrm{NO}$ & 9,7 & Base-fraca & TGl (intestino) \\
\hline
\end{tabular}

No que diz respeito ao diagnóstico, a figura 1 traz as informações sobre o percentual de transtornos mentais apresentados pelos presidiários. Os resultados mostraram que 46,7\% apresentam esquizofrenia paranoide, residual e não-específica, hebefrênica, simplesCID10-F20.0, F20.5, F29.0, F20.1, F20.6 respectivamente; 16,7\% apresentam sicose não-orgânica não-específica (CID10-F29), 19,9\% possuem transtornos mentais e comportamentais com síndrome de dependência, com síndrome amnésica, com transtorno psicótico (CID10-F19.0, F19.5, F19.2, F10.7 respectivamente), epilepsia (G40.3) corresponde a $6,7 \%$ e outros transtornos $23,1 \%$.

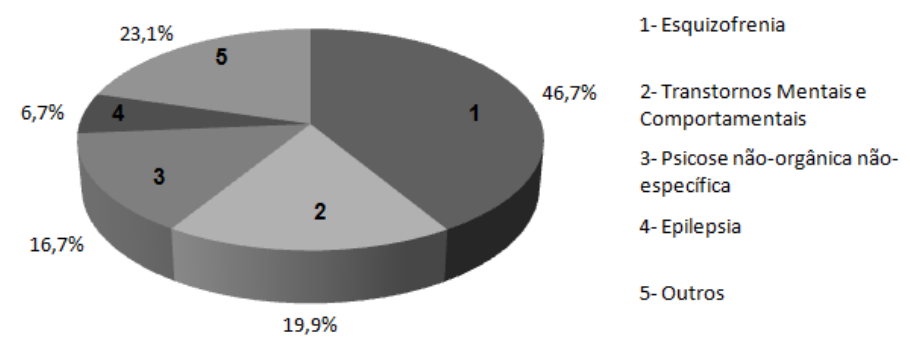

Figura 1. Distribuição da população quanto ao diagnóstico psiquiátrico. 
Outros: Transtorno relacionado ao álcool (F10.9), Transtorno depressivo recorrente (F33.3), Personalidade paranoica (F60.0), Personalidade dissocial (F60.2), Psicose afetiva bipolar (F3.1), Retardo mental (F71.1), Exibicionismo (F65.2).

Encontrou-se que a média do tempo de internação no manicômio é de 11,3 \pm 8,04 anos. Os pacientes que estão cumprindo medida de segurança por homicídio somam 43,29\%. Os outros crimes cometidos incluem: lesão corporal, ameaça, roubo, estupro, atentado violento ao pudor e ato obsceno. A pesquisa buscou investigar se o diagnóstico da esquizofrenia estava diretamente relacionado com o cometimento do homicídio. Foram comparados os índices de homicídio entre os esquizofrênicos e os demais apenados que possuem outros transtornos mentais.

Quanto a prática de homicídio e a existência de patologias mentais, observou-se que os apenados esquizofrênicos que cometeram homicídio somaram 11 casos (36,7\% dos esquizofrênicos); os que apresentam transtornos mentais equivalem a $6,7 \%(n=2)$ dos casos e os presidiários com outros tipos de transtornos mentais equivalem a $23,3 \%$. Ressalta-se que cada presidiário portador de transtornos mentais, destacam-se psicose não-orgânica não específica, transtornos relacionados com o álcool, transtorno depressivo recorrente, personalidade paranoica (tabela 5), cometeram apenas um delito.

Tabela 4. Demonstrativo dos crimes cometidos e tipo de transtorno mental existentes nos detentos.

\begin{tabular}{lrrrrrr}
\hline & \multicolumn{2}{c}{ Homicídio } & \multicolumn{2}{c}{ Lesão Corporal } & \multicolumn{2}{c}{ Roubo } \\
\hline & $\mathrm{f}$ & $\%$ & $\mathrm{f}$ & $\%$ & $\mathrm{f}$ & $\%$ \\
\hline Esquizofrenia & 11 & $36,7 \%$ & 2 & $6,7 \%$ & & - \\
$\begin{array}{l}\text { Transtornos Mentais } \\
\text { e Comportamentais }\end{array}$ & 2 & $6,7 \%$ & & - & 1 & $3,33 \%$ \\
Outros & 7 & $23,3 \%$ & 5 & $10 \%$ & & $26,70 \%$ \\
\hline
\end{tabular}

\section{Discussão}

Os resultados mostram que a amostra estudada é composta, por homens fumantes, na faixa etária de 24 a 66 anos, com baixo nível de escolaridade, constituído, 
predominantemente, por solteiros. Estas características evidenciam desigualdade social construída historicamente e que afeta diretamente a qualidade de vida do paciente (10).

De acordo com o uso dos medicamentos, tem-se que o mais prescrito foi o antipsicótico Haloperidol, dados também obtidos por Marafantiet al (11). Comprovando que a esquizofrenia é o transtorno mental mais prevalente no ambiente penitenciário, sendo o Haloperidol a droga de primeira escolha, havendo uma melhor adesão ao tratamento.Moscatello (12), avaliou 100 pacientes do Manicômio Judiciário Franco da Rocha, onde $44 \%$ deles tiveram diagnóstico de esquizofrenia, dados bem parecidos com os obtidos no presídio Stênio Gomes, sendo que a esquizofrenia equivale 46,7\% dos casos.

A psicose orgânica não-específica aparece em segundo lugar com 13,3\% dos casos, sendo que dois cometeram homicídio qualificado. Foi observado um alto índice de interações medicamentosas neste estudo, visto que estes pacientes estão inseridos no grupo de risco (13). Isto reforça a problemática do tratamento medicamentoso em pacientes criminais, suas consequências e a necessidade de esforços contínuos no sentido de aperfeiçoar a terapêutica farmacológica.

A maioria das potenciais interações medicamentosas identificadas neste estudo apresentou início demorado (74,10\%) de moderada gravidade (51,7\%) e estavam documentadas na literatura como possível (53,43\%). A dispensação dos medicamentos aos internos é realizada pelo pessoal técnico que segue a prescrição. Pela observação in situ percebeu-se que a equipe administra a medicação através da dissolução desta em copos plásticos identificados com o nome de cada paciente, o que garante a adesão e evita erro de medicação.

Estudos recentes têm demonstrado que existe associação entre violência e doença mental (14). Nas ultimas décadas vários estudos sobre associações entre esquizofrenia e homicídios foram realizados, por meio dos registros de documentos médicos e policiais (15). Pesquisa realizada na Inglaterra com presos por crimes violentos verificam maior prevalência de pessoas com esquizofrenia (16). Estes resultados condizem com o que foi avaliado no Instituto Psiquiátrico Stênio Gomes.

Neste contexto, na Dinamarca, Bennanet al. (17) demonstra através de um estudo de coorte em sujeitos de até 44 anos de idade, havendo um total de 358.180 pessoas, que a esquizofrenia era o único transtorno mental a se associar ao aumento no risco de crime 
violento. É atualmente aceito que certos indivíduos com o diagnóstico de esquizofrenia têm significativamente maior probabilidade de exercer um ato violento criminal do que os indivíduos da população geral (18).

Todos os apenados avaliados estavam cumprindo medida de segurança, conceituada como: "uma sanção penal imposta pelo Estado, na execução de uma sentença, cuja finalidade é exclusivamente preventiva, no sentido de evitar que o autor de uma infração penal que tenha demonstrado periculosidade volte a delinquir" (19). Indivíduos portadores de transtornos mentais e/ou psicopatas se enquadram no artigo 26 do Código Penal, possuindo uma pena diminuída, onde o prazo máximo de cumprimento de três anos (20).

Observando que esse ambiente penal não ressocializa esses sujeitos. É importante que as medidas punitivas sejam sempre avaliadas, para quando saírem do ambiente penitenciário possam retornar ao convívio em sociedade (21). Este estudo pôde demonstrar que a realidade de pacientes com transtornos mentais e que cometeram crimes possuem tratamento inadequado, não promovendo uma integração do doente mental à sociedade. As instalações não contribuem para o tratamento, afinal os internos não possuem celas adequadas, boa higienização e muitos são abandonados pelos familiares. Mostram-se ineficazes para os casos que envolvem psicopatas e não conduzem ao objetivo principal, que é ressocializar o indivíduo, e não apenas puni-lo.

\section{Conclusão}

Predominância do gênero masculino, adulto, com baixo grau de escolaridade, são aspectos que se correlacionam com a prática delituosa. A presença de transtornos mentais, particularmente a esquizofrenia, em grau de conduta agressiva, que é um sinal inerente à patologia parece contribuir para a prática de atos violentos.

O homicídio qualificado e outros crimes violentos apresentam uma estreita relação com a esquizofrenia. $\mathrm{O}$ uso de antipsicótico melhora o prognóstico e deve ser garantido a todos que dele necessitem. As interações medicamentosas são reais e podem comprometer a segurança terapêutica. A dispensação adequada associada ao uso racional do medicamento, dentro de um contexto interdisciplinar, pode garantir uma melhora na qualidade de vida do penitenciário portador de patologias psiquiátricas.

A falta de formação profissional diante de um ambiente inóspito e abandono familiar são empecilhos no processo de integração social do sujeito preso. 


\section{Referências}

1. Silva AVBA, Filgueiras RMO. Guia Farmacoterapêutico do Sistema Penitenciário do Estado do Ceará. Secretaria da Justiça e Cidadania, Fortaleza, 2010.

2. Moraes A. Constituição do Brasil Interpretada e legislação Constitucional. São Paulo: Atlas, 2004.

3. Assis RD. As prisões e o direito penitenciário no Brasil: histórico das prisões no Brasil, histórico das leis de execuções penais, aspectos e finalidades da atual lei de execução penal brasileira. Disponível em: http://www.direitonet.com.br/artigos/exibir/3482/As-prisoese-o-direito-penitenciario-no-Brasil Acesso em: 27.7.2011.

4. Organização Mundial de Saúde. Disponível em:

http://www.who.int/entity/whr/2001/en/whr01 en.pdf Acesso em 3.10.2011.

5. Silva MBB. As pessoas em medida de segurança e os hospitais de custódia e tratamento psiquiátrico no contexto do plano nacional de saúde no sistema penitenciário. Rev. Bras. Crescimento desenvolvimento humano, 2010, 20:95-105.

6. Brasil. Portaria n.․ 344, 12 de maio de 1998. Disponível em: Aprova o Regulamento Técnico sobre substâncias e medicamentos sujeitos a controle especial. http://bvsms.saude.gov.br/bvs/saudelegis/svs/1998/prt0344 12051998 rep.html Acesso em: 1.11.2011.

7. González OV, Donoso IA, Blanco MG. Uso de Psicofármacosenprisión (CP Madrid III). Rer. Esp. SanidPenit, Madrid, 2007, 9:38-46.

8. Lindqvist P, Allebeck P. Schizophenia and crime. A longitudinal follow-up of 644 schizophrenics in Stockholm.Br J. Psychiaty, 1990, 50:157:345.

9. Tatro DS et al. Drug Interaction Facts.St. Louis: Wolters Kluwer, 2010.

10. Cardoso CS et al. Qualidade de vida e dimensão ocupacional na esquizofrenia: uma comparação por sexo. Cad. Saúde Pública, Rio de Janeiro, 2006, 22(6):1303-1314.

11. Marafanti í et al. Características da População carcerária atendida no serviço de urgência psiquiátrica do Centro de Atenção Integrada á Saúde mental da Santa Casa de Misericórdia de São. Rev. de Criminologia e Ciências Penitenciárias, São Paulo, 2012.

12. Moscastello R. Comparação entre diagnóstico psiquiátrico e delito cometido em 100 pacientes do Manicômio Judiciário de Franco da Rocha. Rev. Bras. Psiquiatria, 1999, $21: 131$. 
13. Secoli SR. Interações medicamentosas: fundamentos para a prática clínica da enfermagem. Disponível em:

http://www.ee.usp.br/reeusp/upload/html/560/body/v35n1a04.htm Acesso em 20.10.2012.

14. COID J. Dangerous patients with mental illness: increased risks warrant new policies, adequate resources and appropriate legislation. BMJ, 1996, 6:312-965.

15. Asnis GM, Kaplan ML, Hundorfean SAEED. Violence and homicidal behaviors in psychiatric disorders. PsychiatricClinicsofNorth America, 1997, 20(2):405-25.

16. Taylor PJ, Gunn JC. Violence and psychosis. I. Risk of violence among psychotic men. BMJ (Clin Res Ed), 1984, 9:288-1945.

17. Brennan PA, Mednick SA, Hodgins S. Major mental disorders and criminal violence in a Danish birth cohort. Archivesof General Psychiatry, 2000, 57:494-500.

18. Eronen M, Hakola $\mathrm{P}$, Tiihonen J. Mental disorders and homicidal behavior in Finland.ArchGenPsychiatry, 1996, 53:497-501.

19. Capez F. Curso de direito penal: parte geral. São Paulo: Saraiva, 2005.

20. Brasil. Tribunal de Justiça. Terceira Câmara Criminal. Apelação Crime no 70037449089. Relator: OdoneSanguiné. Julgado em: 17 mar. 2011.

21. Castro IM de. Psicopatia e suas consequências jurídico-penais. Disponível em: http://www3.pucrs.br/pucrs/files/uni/poa/direito/graduacao/tcc/tcc2/trabalhos2012 1/isabel castro.pdf Acesso em 18.10.2012.

Recebido em: 15.5 .2018

Aprovado em: 11.6.2018

\section{Como citar este artigo:}

Almeida AP, Lima RP, Morais ACLN. Análise do uso de medicamentos psicotrópicos no sistema penitenciário do Estado do Ceará. Revista Cadernos Ibero-Americanos de Direito Sanitário. 2018 abr./jun, 7(2):82-94. 